\title{
Opportunistic Secrecy with a Strict Delay Constraint
}

\author{
Karim Khalil, O. Ozan Koyluoglu, Hesham El Gamal, and Moustafa Youssef
}

\begin{abstract}
We investigate the delay limited secrecy capacity of the flat fading channel under two different assumptions on the available transmitter channel state information (CSI). The first scenario assumes perfect prior knowledge of both the main and eavesdropper channel gains. Here, upper and lower bounds on the delay limited secrecy capacity are derived, and shown to be tight in the high signal-to-noise ratio (SNR) regime. In the second scenario, only the main channel CSI is assumed to be available at the transmitter where, remarkably, we establish the achievability of a non-zero delay-limited secure rate, for a wide class of channel distributions, with a high probability. In the two cases, our achievability arguments are based on a novel two-stage key-sharing approach that overcomes the secrecy outage phenomenon observed in earlier works.
\end{abstract}

\section{INTRODUCTION}

Many wireless applications are limited by different forms of delay constraints. These applications range from the most basic voice communication to the more demanding multimedia streaming. However, due to its broadcast nature, the wireless channel is vulnerable to eavesdropping and other security threats. Therefore, techniques that satisfy both the delay limitation and the confidentiality requirement are of definite interest. This motivates our analysis of the fundamental (information theoretic) limits of secure communication over fading channels subject to strict deadlines.

Recent works on information theoretic security have been largely inspired by the wire-tap channel model of Wyner [1]. In this seminal work, Wyner established the achievability of non-zero secrecy capacity when the wiretapper channel is a degraded version of the main one, by exploiting the noise to create an advantage for the legitimate receiver. More recently, the effect of fading on the secrecy capacity was studied in [2] in the ergodic setting. The main insight offered by this work is the achievability of a non-zero secrecy capacity, by opportunistically

This work is submitted to the IEEE Transactions on Information Theory.

Karim Khalil and Moustafa Youssef are with the Wireless Intelligent Networks Center (WINC), Nile University, Cairo, Egypt. Email: \{kareem.makarem,mayoussef\}@nileu.edu.eg. O. Ozan Koyluoglu and Hesham El Gamal are with the Department of Electrical and Computer Engineering, The Ohio State University, Columbus, OH 43210 USA. Email: \{koyluogo,helgamal\}@ece.osu.edu.

This work is supported in part by an NPRP grant from the QNRF, the Egyptian NTRA, and the National Science Foundation (NSF). The material in this paper was presented in part at the IEEE International Symposium on Information Theory, Seoul, Korea in July 2009. 
exploiting the multi-path channel fluctuations, even when the eavesdropper channel is better than the legitimate one on the average.

On the other side, delay limited transmission over fading channels has been well studied in different network settings and using various traffic models. For example, in [3], the delay limited capacity notion was introduced and the optimal power control policies were characterized in several interesting scenarios. In [4], the strict delay limitation of [3] was relaxed by allowing for buffering the packets at the transmitter. In this setup, the asymptotic behavior of the power-delay trade-off curve was characterized yielding valuable insights on the structure of the optimal resource allocation strategies [4]. More recently, the scheduling problem of data transmission over a finite delay horizon assuming perfect CSI was considered in [5]. Our work can be viewed as a generalization of [3] whereby a secrecy constraint is imposed on the problem.

The delay limited transmission of secure data over fading channels was considered previously in [6]. In that work, the authors attempted to send the secure information using binning techniques inspired by the wiretap channel results. The drawback of this approach is that it fails to secure the information in the particular instants where the eavesdropper channel gain is larger than that of the main channel. This results in the so-called secrecy outage phenomenon (as defined in [6]). Unfortunately, in the delay limited setting, the secrecy outage can not be made to vanish by increasing the transmission power, since it does not offer a relative advantage to the legitimate receiver, leading to the conclusion that the delay limited secure rate achieved by this approach is equal to zero for most channel distributions of interest [6]. This obstacle is overcome by our two-stage approach. Here, the delay sensitive data is secured via Vernam's one time pad approach [7] (see also [8]) using a private key, which was shared secretly by the two legitimate nodes during previous transmissions. Since the key packets are not delay sensitive, the two nodes can share the key by distributing its bits over many fading realizations to capitalize on the ergodic behavior of the channel. Our result is enabled by observing that, through the appropriate rate allocation, the key bits can be superimposed on the delay sensitive data packets so that they can be used for securing future packets. This mechanism is referred as key renewal process in the sequel. This process requires an initialization phase to share the key needed for securing the first data packets. However, the loss in throughput entailed by the initialization overhead vanishes in the asymptotic limit of a large number of data packets. Our analytical results establish the asymptotic optimality, with high SNR, of this novel approach in the scenario where both the main and eavesdropper channel gains are known a-priori at the transmitter. When only the main channel CSI is available, this approach is shown to achieve a non-zero constant secure rate for a wide class of quasi-static and invertible channels [3] with high probability ${ }^{1}$.

The rest of the paper is organized as follows. Section II introduces the system model and notations used throughout the paper. Section III focuses on the full CSI scenario whereas the case with only the main channel CSI is analyzed in Section IV; along with some representative numerical results. Finally, some concluding remarks are offered in

\footnotetext{
${ }^{1}$ We use a modified version of the $\epsilon$-achievable rate defined in [9] to argue that our results are achievable with a vanishing probability of secrecy outage.
} 
Section V.

\section{SYSTEM MODEL}

The system model is shown in Figure 1. A source node (Alice) wishes to communicate with a destination node (Bob) over a fading channel in the presence of an eavesdropper (Eve). We adopt a block fading model, in which the channel is assumed to be constant during a coherence interval and changes randomly from an interval to the next according to a bounded continuous distribution. Also, the coherence intervals are assumed to be large enough to allow for the use of random coding arguments. During any symbol interval $i$, the signals received at the destination and the eavesdropper, respectively, are given by

$$
\begin{aligned}
& y(i)=g_{m}(i) x(i)+w_{m}(i), \\
& z(i)=g_{e}(i) x(i)+w_{e}(i),
\end{aligned}
$$

where $x(i)$ is the transmitted symbol, $g_{m}(i)$ and $g_{e}(i)$ are the main channel and the eavesdropper channel gains respectively, $w_{m}(i)$ and $w_{e}(i)$ are the i.i.d. additive white complex gaussian noise with unit variance at the legitimate receiver and the eavesdropper, respectively. We denote the power gains of the fading channels for the main and eavesdropper channels by $h_{m}(i)=\left|g_{m}(i)\right|^{2}$ and $h_{e}(i)=\left|g_{e}(i)\right|^{2}$, respectively. We impose the long term average power constraint $\bar{P}$, i.e.,

$$
\mathbb{E}[P(\mathbf{h})] \leq \bar{P}
$$

where $P(\mathbf{h})$ is the power allocated for the channel state $\mathbf{h}=\left(h_{m}, h_{e}\right)$ and the expectation is over the channel gains.

The source wishes to send a message $W \in \mathcal{W}=\{1,2, \cdots, M\}$ to the destination while satisfying the delay and secrecy constraints. In the following, our delay constraint is imposed by breaking our message into packets of equal sizes, where each one is encoded independently, transmitted in only one coherence block, and decoded by the main receiver at the end of this block. The total transmission time of $n$ channel symbol durations is divided into coherence intervals of length $n^{\prime}$ channel uses; for which both $h_{m}$ and $h_{e}$ remain fixed. We assume there are total of $S B$ number of such fading blocks. These blocks are grouped into $S$ super-blocks, each consisting of $B$ fading blocks. We will further represent a fading block with tuple $(s, b)$ such that $s \in\{1,2, \cdots, S\}$ denotes the super-block index and $b \in\{1,2, \cdots, B\}$ denotes the fading block index within a particular super-block. We consider the problem of constructing $\left(M^{\prime}, n^{\prime}\right)$ codes $\left(M=S B M^{\prime}\right)$ to transmit the message of the block $(s, b)$, which is represented by $W(s, b) \in \mathcal{W}^{\prime}=\left\{1,2, \ldots, M^{\prime}\right\}$, to the receiver. Here, an $\left(M^{\prime}, n^{\prime}\right)$ code consists of the following elements: 1) a stochastic encoder $f_{n^{\prime}}($.$) at the source that maps the message w(s, b)$ to a codeword $X^{n^{\prime}}(s, b) \in \mathcal{X}^{n^{\prime}}$, and 2) a decoding function $\phi: \mathcal{Y}^{n^{*}} \rightarrow \mathcal{W}^{\prime}$ at the legitimate receiver, where $n^{*}=(s-1) B n^{\prime}+b n^{\prime}$ denotes the total number of the received signal dimension at the receiver at the end of the block $(s, b)$. The average error probability of an $\left(M^{\prime}, n^{\prime}\right)$ code is defined as

$$
P_{e}^{n^{\prime}}(s, b)=\frac{1}{M^{\prime}} \sum_{w \in \mathcal{W}^{\prime}} \operatorname{Pr}\left(\left\{\phi\left(y^{n^{*}}\right) \neq w \mid w \text { is sent in block }(s, b)\right\}\right),
$$


where $y^{n^{*}}$ represents the total received signals at the legitimate receiver at the end of the block $(s, b)$. We define the equivocation rate $R_{e}$ at the eavesdropper as the entropy rate of the transmitted message over block $(s, b)$ conditioned on the available CSI and all the channel outputs at the eavesdropper, i.e.,

$$
R_{e}(s, b) \triangleq \frac{1}{n^{\prime}} H\left(W(s, b) \mid Z^{n}, h_{m}^{n}, h_{e}^{n}\right),
$$

where $h_{m}^{n}=\left\{h_{m}(1), \cdots, h_{m}(n)\right\}$ and $h_{e}^{n}=\left\{h_{e}(1), \cdots, h_{e}(n)\right\}$ denote the channel power gains of the legitimate receiver and the eavesdropper in $n$ symbol intervals, respectively. We consider only the perfect secrecy (in the sense of [1]) which requires the equivocation rate $R_{e}$ to be arbitrarily close to the message rate. Hence, we define the achievability of the delay limited secrecy rate and capacity, respectively, as follows.

Definition 1: The rate $R_{s, d}$ is said to be an achievable delay limited secrecy rate, if for any $\epsilon^{\prime}>0$, there exist a $\left(2^{n^{\prime} R_{s, d}}, n^{\prime}\right)$ code such that

$$
\begin{aligned}
P_{e}^{n^{\prime}}(s, b) & \leq \epsilon^{\prime}, \\
R_{e}(s, b) & \geq R_{s, d}-\epsilon^{\prime}
\end{aligned}
$$

for every fading block $(s, b), s \neq 1$, and for sufficiently large $n, B$. The delay limited secrecy capacity, $C_{s, d}$, is defined as the supremum of the achievable delay limited perfect secrecy rates.

Here, if the secrecy constraint (5) is not satisfied for a given block, then the corresponding block is said to be in secrecy outage, the probability of which is defined as follows.

Definition 2: For a given $\epsilon^{\prime}>0$, the probability of secrecy outage for the block $(s, b)$ evaluated at rate $R_{s, d}$ is given by

$$
P_{\text {out }}\left(s, b, R_{s, d}, \epsilon^{\prime}\right) \triangleq \operatorname{Pr}\left(\left\{R_{e}(s, b) \leq R_{s, d}-\epsilon^{\prime}\right\}\right) .
$$

Now, we define a modified version of the $\epsilon$-achievable rate notion given by [9] (see also [10]) for the secrecy outage phenomenon.

Definition 3: The rate $R_{s, d}(\epsilon)$ is said to be an $\epsilon$-achievable delay limited secrecy rate, if for any $\epsilon^{\prime}>0$, there exists a $\left(2^{n^{\prime} R_{s, d}(\epsilon)}, n^{\prime}\right)$ code such that

$$
\begin{aligned}
P_{e}^{n^{\prime}}(s, b) & \leq \epsilon^{\prime} \\
P_{\text {out }}\left(s, b, R_{s, d}(\epsilon), \epsilon^{\prime}\right) & \leq \epsilon
\end{aligned}
$$

for every fading block $(s, b), s \neq 1$, and for sufficiently large $n, B$. The $\epsilon$-delay limited secrecy capacity, $C_{s, d}(\epsilon)$, is defined as the supremum of the achievable delay limited secrecy rates with secrecy outage probability less than $\epsilon$.

We note that in our achievability results, an initialization phase occurs during the first super-fading block $(s=1)$, and its duration is negligible as $S \rightarrow \infty$. This explains why the requirements of Definitions 1 and 3 are satisfied for every fading block $(s, b)$ with $s \neq 1$.

Finally, we give some notational remarks. We denote the delay limited secrecy rate and capacity as $R_{s, d}^{F}, C_{s, d}^{F}$, respectively, for the full CSI scenario, where both $g_{m}$ and $g_{e}$ are known a-priori at the transmitter. For the main 
CSI scenario, where only $g_{m}$ is known a-priori at the transmitter, the delay limited secrecy rate, secrecy capacity, $\epsilon$-achievable secrecy rate, and $\epsilon$-secrecy capacity, are denoted respectively by $R_{s, d}^{M}, C_{s, d}^{M}, R_{s, d}^{M}(\epsilon)$ and $C_{s, d}^{M}(\epsilon)$. We let $[x]^{+}=\max \{x, 0\} \cdot \log (\cdot)$ denotes the base-2 logarithm. Throughout the sequel, the expectations are taken with respect to the random channel gains.

\section{Full TRAnsmitter CSI}

First, we give a simple upper bound on the delay limited secrecy capacity. This bound will be used to establish the optimality of the proposed two-stage approach in the high SNR regime.

Theorem 1: The delay limited secrecy capacity when both $g_{m}$ and $g_{e}$ are available at the transmitter, $C_{s, d}^{F}$, is upper bounded by

$$
C_{s, d}^{F} \leq \max _{\substack{P(\mathbf{h}) \\ \text { s.t. } \mathbb{E}[P(\mathbf{h})] \leq \bar{P}}} \min \left\{R_{s}^{F}, R_{d}^{F}\right\},
$$

where $R_{s}^{F}$ and $R_{d}^{F}$ are given as follows.

$$
\begin{aligned}
R_{s}^{F} & =\mathbb{E}\left[\log \left(1+P(\mathbf{h}) h_{m}\right)-\log \left(1+P(\mathbf{h}) h_{e}\right)\right]^{+} \\
R_{d}^{F} & =\min _{\mathbf{h}} \log \left(1+P(\mathbf{h}) h_{m}\right)
\end{aligned}
$$

Proof: Consider an arbitrary power allocation scheme $P(\mathbf{h})$. Since imposing delay constraint can only degrade the performance, we upper bound the achievable delay limited secrecy rate with the ergodic secrecy rate as

$$
R_{s, d}^{F} \leq R_{s}^{F}
$$

We also have

$$
R_{s, d}^{F} \leq R_{d}^{F}
$$

since imposing the secrecy constraint can not increase the achievable rate. Then, combining (9) and (10), and maximizing over $P(\mathbf{h})$, we obtain

$$
R_{s, d}^{F} \leq \max _{P(\mathbf{h})} \min \left\{R_{d}^{F}, R_{s}^{F}\right\}
$$

which proves our claim.

The following result establishes a lower bound on the delay limited secrecy capacity using our novel twostage approach. The key idea is to share a private key between Alice and Bob, without being constrained by the delay limitation. This key is, then, used to secure the delay sensitive data while overcoming the secrecy outage phenomenon. In the steady state, the key renewal process takes place by superimposing the key on the delay sensitive traffic. More precisely, as outlined in the proof, the delay sensitive traffic (secured by the previously shared key) serves as a randomization signal in the binning scheme used to secure the current key. Finally, since $h_{e}$ is known a-priori at the transmitter, one can further increase the delay limited secrecy rate by dedicating a portion of the secure rate to the delay sensitive traffic (as controlled by the function $q(\mathbf{h})$ in the following theorem). 
Theorem 2: The delay limited secrecy capacity in the full CSI scenario, $C_{s, d}^{F}$, is lower bounded by the following achievable rate.

$$
C_{s, d}^{F} \geq R_{s, d}^{F}=\max _{\substack{P(\mathbf{h}), q(\mathbf{h}) \\ \text { s.t. } \mathbb{E}[P(\mathbf{h})] \leq \bar{P}}}\left[\min _{\mathbf{h}}\left\{R_{1}(\mathbf{h})+R_{2}(\mathbf{h})\right\}\right]
$$

where

$$
\begin{aligned}
R_{s}(\mathbf{h}) & =\left[\log \left(1+P(\mathbf{h}) h_{m}\right)-\log \left(1+P(\mathbf{h}) h_{e}\right)\right]^{+} \\
R_{k}(\mathbf{h}) & =\left[\log \left(1+P(\mathbf{h}) h_{m}\right)-\log (1+P(\mathbf{h}) q(\mathbf{h}))\right]^{+} \\
R_{2}(\mathbf{h}) & =R_{s}(\mathbf{h})-R_{k}(\mathbf{h}),
\end{aligned}
$$

$q(\mathbf{h}) \geq h_{e}, \forall h_{e}$, and $R_{1}(\mathbf{h})$ is chosen to satisfy the following

$$
\begin{aligned}
\mathbb{E}\left[R_{1}(\mathbf{h})\right] & \leq \mathbb{E}\left[R_{k}(\mathbf{h})\right] \\
R_{1}(\mathbf{h}) & \leq \min \left\{\log \left(1+P(\mathbf{h}) h_{m}\right), \log \left(1+P(\mathbf{h}) h_{e}\right)\right\}
\end{aligned}
$$

Proof: Consider a fixed pair $\left(h_{m}, h_{e}\right)$, a power control policy $P(\mathbf{h})$ satisfying $E[P(\mathbf{h})] \leq \bar{P}$, and an arbitrary function $q(\cdot)$ such that $q(\mathbf{h}) \geq h_{e}$. The achievable rate is obtained by finding the minimum rate over the pair $\left(h_{m}, h_{e}\right)$, to satisfy our strict delay constraint, and then maximizing over all power control policies and functions $q(\mathbf{h})$. We start the proof by defining the different rates in (12), (13), (14): $R_{s}(\mathbf{h})$ is the instantaneous secrecy rate supported by the channel, $R_{k}(\mathbf{h})$ is the rate used to share the private key, $R_{2}(\mathbf{h})$ is the delay limited secrecy rate of the data that is transmitted without the key, and $R_{1}(\mathbf{h})$ is the rate of the data sent via the one time pad scheme. Moreover, we define the additional randomization rate by

$$
R_{x}(\mathbf{h})=\min \left\{\log \left(1+P(\mathbf{h}) h_{m}\right), \log \left(1+P(\mathbf{h}) h_{e}\right)\right\}-R_{1}(\mathbf{h})
$$

Our Two-stage Scheme: We divide the message $W \in \mathcal{W}=\left\{1,2, \cdots, 2^{n R_{s, d}^{F}}\right\}$ into $(S-1) B$ data packets $D(s, b)$, each encoded independently and sent with rate $R_{s, d}^{F}$ during the block of the channel where $s \in\{2, \cdots, S\}$ and $b \in\{1,2, \cdots, B\}$. We further divide each data packet into two parts: $\tilde{D}_{1}(s, b)$ which is sent as an open message (after being encrypted by the key) and $D_{2}(s, b)$ which is sent as a secure message. Our scheme uses a separation strategy similar to [11] by sending public and private messages simultaneously. But in contrast to [11], we exploit the fading channel to secure the key, and hence, the message. We now describe the initial key generation and key renewal processes. For the very first $B$ blocks (the super-block $s=1$ ), we generate random key bits, $K(1)$, and then transmit them from Alice to Bob securely. Utilizing the ergodicity of the channel, we can transmit a key of an approximate length $n^{\prime} B \mathbb{E}\left[R_{k}(\mathbf{h})\right]$ bits [2]. Then, for any super-block $s>1$, we will use the key $K(s-1)$ for the one time pad, and also generate a new key $K(s)$ for the use in the next super-block. Here, to secure the open packet of block $(s, b)$, we use $n^{\prime} R_{1}(\mathbf{h})$ bits from the remaining bits of the key $K(s-1)$, represented by $\tilde{K}(s, b)$, to encrypt the data packet $\tilde{D}_{1}(s, b)$ using one time pad encryption:

$$
D_{1}(s, b)=\tilde{D}_{1}(s, b) \oplus \tilde{K}(s, b) .
$$


The encoder will declare an encoding error, if there are not sufficient key bits left in $K(s-1)$ for the one time pad encryption. To summarize, during the block $(s, b)$, four messages are combined together and sent over the channel:

1) $D_{1}(s, b)$ is mapped into $W_{1}(s, b) \in \mathcal{W}_{1}=\left\{1,2, \cdots, 2^{n^{\prime} R_{1}(\mathbf{h})}\right\}$.

2) $D_{2}(s, b)$ is mapped into $W_{2}(s, b) \in \mathcal{W}_{2}=\left\{1,2, \cdots, 2^{n^{\prime} R_{2}(\mathbf{h})}\right\}$.

3) The key bits $D_{k}(s, b)$ are mapped into $W_{k}(s, b) \in \mathcal{W}_{k}=\left\{1,2, \cdots, 2^{n^{\prime} R_{k}(\mathbf{h})}\right\}$.

4) Additional randomization is mapped into $W_{x}(s, b) \in \mathcal{W}_{x}=\left\{1,2, \cdots, 2^{n^{\prime} R_{x}(\mathbf{h})}\right\}$.

Codebook Generation and Encoding: Our random coding arguments rely on an ensemble of codebooks generated according to a zero-mean Gaussain distribution with variance $P(\mathbf{h})$. If there are enough number of key bits for the one time pad scheme (i.e., no encoding error), the encoder will work as follows. For a given block $(s, b)$, let $R=\log \left(1+P(\mathbf{h}) h_{m}(s, b)\right)-\epsilon$. When $h_{m}(s, b) \leq h_{e}(s, b)$, we have one of $2^{n^{\prime}\left(R_{1}(\mathbf{h})+R_{x}(\mathbf{h})\right)}$ open messages, denoted by the pair $\left(w_{1}, w_{x}\right)$, to be sent. To encode the message $\left(w_{1}, w_{x}\right)$, the encoder selects the codeword $X^{n^{\prime}}\left(w_{1}, w_{x}\right)$ from the chosen codebook. On the other hand, when $h_{m}(s, b) \geq h_{e}(s, b)$, a binning scheme (see, e.g., [1]) is used to send secret bits over the channel. We first generate a Gaussian codebook consisting of $2^{n^{\prime} R}$ codewords, represented by $X^{n^{\prime}}$, and then independently assign each of them to one of $2^{n^{\prime}\left(R_{k}(\mathbf{h})+R_{2}(\mathbf{h})\right)}$ bins, where the bin index is $\left(w_{k}, w_{2}\right)$, according to a uniform distribution. This ensures that any of the sequences are equally likely to be within any of the bins. Each bin has $2^{n^{\prime}\left(R_{1}(\mathbf{h})+R_{x}(\mathbf{h})\right)}$ sequences with codeword index denoted by $\left(w_{1}, w_{x}\right)$. Accordingly, a sequence is represented by the tuple of indices $\left(w_{k}, w_{2}, w_{1}, w_{x}\right)$. To encode a particular key-message pair, the encoder chooses a codeword indexed by $\left(w_{1}, w_{x}\right)$ from the bin indexed by $\left(w_{k}, w_{2}\right)$, i.e., $X^{n^{\prime}}\left(w_{k}, w_{2}, w_{1}, w_{x}\right)$, and send it over the channel. We note that $w_{x}$ is uniformly chosen among $\mathcal{W}_{x}$ and $w_{1}$ is determined by the data $\tilde{D}_{1}(s, b)$ and the corresponding key bits of the previous super-block $\tilde{K}(s, b)$, and hence uniformly distributed over $\mathcal{W}_{1}$.

Error Analysis: For each fading block $(s, b)$, we denote the encoding and decoding error events by $E_{\text {enc }}(s, b)$ and $E_{\mathrm{dec}}(s, b)$, respectively. Then, we write the error probability at the receiver as follows.

$$
P_{e}^{n^{\prime}}(s, b)=\operatorname{Pr}\left\{E_{\mathrm{enc}}(s, b)\right\}+\operatorname{Pr}\left\{E_{\mathrm{dec}}(s, b) \mid E_{\mathrm{enc}}^{c}(s, b)\right\}
$$

Since we only impose a constraint on $\mathbb{E}\left[R_{1}(\mathbf{h})\right]$ in (14), there will be a non-zero probability that the key bits fall short. In such a case the encoder will declare an error. Hence, we can write the following bound.

$$
\operatorname{Pr}\left\{E_{\text {enc }}(s, b)\right\} \leq \operatorname{Pr}\left\{\sum_{b=1}^{B} R_{1}(s, b)>\sum_{b=1}^{B} R_{k}(s-1, b)\right\}
$$

Here, from the strong law of large numbers and from (14), we see that the right hand side of (18) and hence $\operatorname{Pr}\left\{E_{\text {enc }}(s, b)\right\}$ can be arbitrarily made small as $B \rightarrow \infty$.

Now, it remains to show that $\operatorname{Pr}\left\{E_{\mathrm{dec}}(s, b) \mid E_{\mathrm{enc}}^{c}(s, b)\right\}$ can be arbitrarily made small. This follows as $n^{\prime} \rightarrow \infty$, by applying the asymptotic equipartition property and jointly typical decoding [12]. In particular, for $h_{m}(s, b) \leq$ $h_{e}(s, b)$, the messages $w_{1}$ and $w_{x}$; and for $h_{m}(s, b) \geq h_{e}(s, b)$, the messages $w_{1}, w_{x}, w_{k}$, and $w_{2}$ can be transmitted reliably. Furthermore, as $B \rightarrow \infty$, the average key rate $\mathbb{E}\left[R_{k}(\mathbf{h})\right]$ is achievable within any super-block [2]. 
Equivocation Computation: Here, we show that the secrecy condition given by (5) is satisfied for each fading block $(s, b), s>1$. We can write

$$
\begin{aligned}
n^{\prime} R_{e}(s, b) \stackrel{(a)}{=} & H\left(\tilde{D}_{1}(s, b), D_{2}(s, b) \mid Z^{n}, h_{m}^{n}, h_{e}^{n}\right) \\
= & H\left(\tilde{D}_{1}(s, b), D_{2}(s, b) \mid Z^{n^{\prime}}(1,1), Z^{n^{\prime}}(1,2), \cdots, Z^{n^{\prime}}(s, b), h_{m}^{n}, h_{e}^{n}\right) \\
\stackrel{(b)}{=} & H\left(\tilde{D}_{1}(s, b), D_{2}(s, b) \mid Z^{n^{\prime}}(s, b), Z^{B n^{\prime}}(s-1), h_{m}^{n}, h_{e}^{n}\right) \\
= & H\left(D_{2}(s, b) \mid Z^{n^{\prime}}(s, b), Z^{B n^{\prime}}(s-1), h_{m}^{n}, h_{e}^{n}\right) \\
& +H\left(\tilde{D}_{1}(s, b) \mid D_{2}(s, b), Z^{n^{\prime}}(s, b), Z^{B n^{\prime}}(s-1), h_{m}^{n}, h_{e}^{n}\right)
\end{aligned}
$$

where $Z^{B n^{\prime}}(s-1)=Z^{n^{\prime}}(s-1,1), Z^{n^{\prime}}(s-1,2), \cdots, Z^{n^{\prime}}(s-1, B)$ is the output of the channel at the eavesdropper in the previous super-block $s-1$, (a) follows from splitting the data $D(s, b)$ into the two parts $\tilde{D}_{1}(s, b)$ and $D_{2}(s, b)$, and (b) follows from the independence between block $(s, b)$ and other received signals at the eavesdropper. We now focus on the first term in (19). We note that, in the case where $h_{m}<h_{e}$, no secret bits are sent and hence the first term is zero. When $h_{m}>h_{e}$, in addition to $W_{x}(s, b)$, we use the data $D_{1}(s, b)$ as a randomization signal to secure the messages $D_{2}(s, b)$ and $D_{k}(s, b)$. In this case, the first term in (19) can be lower bounded by the following two steps. First,

$$
\begin{aligned}
\frac{1}{n^{\prime}} H\left(D_{2}(s, b), D_{k}(s, b) \mid Z^{n^{\prime}}(s, b), Z^{B n^{\prime}}(s-1), h_{m}^{n}, h_{e}^{n}\right) & \stackrel{(a)}{=} \frac{1}{n^{\prime}} H\left(D_{2}(s, b), D_{k}(s, b) \mid Z^{n^{\prime}}(s, b), h_{m}^{n}, h_{e}^{n}\right) \\
& \stackrel{(b)}{\geq} \frac{1}{n^{\prime}} H\left(D_{2}(s, b), D_{k}(s, b)\right)-\epsilon \\
& \stackrel{(c)}{=} \frac{1}{n^{\prime}} H\left(D_{2}(s, b)\right)+H\left(D_{k}(s, b)\right)-\epsilon
\end{aligned}
$$

where (a) follows from the independence of $\left(D_{2}(s, b), D_{k}(s, b)\right)$ and the previous super-block, (b) is a result of using the scheme in [2] and the results of [1], i.e., the secrecy of $D_{2}(s, b)$ and $D_{k}(s, b)$, along with the appropriate choice of the randomization rate such that $R_{1}(\mathbf{h})+R_{x}(\mathbf{h})=I(X(s, b) ; Z(s, b))$ and (c) follows from the independence of $D_{2}(s, b)$ and $D_{k}(s, b)$. Second, from (20), we have

$$
\frac{1}{n^{\prime}}\left(H\left(D_{2} \mid Z^{n^{\prime}}, Z^{B n^{\prime}}(s-1), h_{m}^{n}, h_{e}^{n}\right)+H\left(D_{k} \mid D_{2}, Z^{n^{\prime}}, Z^{B n^{\prime}}(s-1), h_{m}^{n}, h_{e}^{n}\right)\right) \geq \frac{1}{n^{\prime}} H\left(D_{2}\right)+\frac{1}{n^{\prime}} H\left(D_{k}\right)-\epsilon,
$$

implying

$$
\begin{aligned}
\frac{1}{n^{\prime}} H\left(D_{2} \mid Z^{n^{\prime}}, Z^{B n^{\prime}}(s-1), h_{m}^{n}, h_{e}^{n}\right) & \geq \frac{1}{n^{\prime}} H\left(D_{2}\right)+\frac{1}{n^{\prime}} I\left(D_{k} ; D_{2}, Z^{n^{\prime}}, Z^{B n^{\prime}}(s-1), h_{m}^{n}, h_{e}^{n}\right)-\epsilon \\
& \geq \frac{1}{n^{\prime}} H\left(D_{2}\right)-\epsilon
\end{aligned}
$$

where we have dropped the index $(s, b)$ for simplicity of notation and the last inequality follows from the fact that mutual information is non-negative. 
The second term in (19) is lower bounded as

$$
\begin{aligned}
\frac{1}{n^{\prime}} H\left(\tilde{D}_{1} \mid D_{2}, Z^{n^{\prime}}, Z^{B n^{\prime}}(s-1), h_{m}^{n}, h_{e}^{n}\right)= & \frac{1}{n^{\prime}} H\left(\tilde{D}_{1} \mid Z^{B n^{\prime}}(s-1), h_{m}^{n}, h_{e}^{n}\right) \\
& -\frac{1}{n^{\prime}} I\left(\tilde{D}_{1} ; Z^{n^{\prime}}, D_{2} \mid Z^{B n^{\prime}}(s-1), h_{m}^{n}, h_{e}^{n}\right) \\
= & \frac{1}{n^{\prime}} H\left(\tilde{D}_{1} \mid h_{m}^{n}, h_{e}^{n}\right) \\
& -\frac{1}{n^{\prime}} I\left(\tilde{D}_{1} ; Z^{n^{\prime}} \mid Z^{B n^{\prime}}(s-1), D_{2}, h_{m}^{n}, h_{e}^{n}\right)
\end{aligned}
$$

since $\tilde{D}_{1}$ is independent of $Z^{B n^{\prime}}(s-1)$ and $D_{2}$. The second term in (22) is upper bounded as

$$
\begin{aligned}
\frac{1}{n^{\prime}} I\left(\tilde{D}_{1} ; Z^{n^{\prime}} \mid Z^{B n^{\prime}}(s-1), D_{2}, h_{m}^{n}, h_{e}^{n}\right) \leq & \frac{1}{n^{\prime}} I\left(\tilde{D}_{1} ; Z^{n^{\prime}}, D_{1} \mid Z^{B n^{\prime}}(s-1), D_{2}, h_{m}^{n}, h_{e}^{n}\right) \\
= & \frac{1}{n^{\prime}} H\left(\tilde{D}_{1} \mid Z^{B n^{\prime}}(s-1), D_{2}, h_{m}^{n}, h_{e}^{n}\right) \\
& -\frac{1}{n^{\prime}} H\left(\tilde{D}_{1} \mid Z^{n^{\prime}}, D_{1}, Z^{B n^{\prime}}(s-1), D_{2}, h_{m}^{n}, h_{e}^{n}\right) \\
\stackrel{(a)}{=} & \frac{1}{n^{\prime}} H\left(\tilde{D}_{1} \mid Z^{B n^{\prime}}(s-1), h_{m}^{n}, h_{e}^{n}\right) \\
& -\frac{1}{n^{\prime}} H\left(\tilde{D}_{1} \mid D_{1}, Z^{B n^{\prime}}(s-1), h_{m}^{n}, h_{e}^{n}\right) \\
= & \frac{1}{n^{\prime}} I\left(\tilde{D}_{1} ; D_{1} \mid Z^{B n^{\prime}}(s-1), h_{m}^{n}, h_{e}^{n}\right) \\
= & \frac{1}{n^{\prime}} H\left(D_{1} \mid Z^{B n^{\prime}}(s-1), h_{m}^{n}, h_{e}^{n}\right) \\
& -\frac{1}{n^{\prime}} H\left(D_{1} \mid \tilde{D}_{1}, Z^{B n^{\prime}}(s-1), h_{m}^{n}, h_{e}^{n}\right) \\
= & \frac{1}{n^{\prime}} H\left(\tilde{D}_{1} \oplus \tilde{K} \mid Z^{B n^{\prime}}(s-1), h_{m}^{n}, h_{e}^{n}\right) \\
& -\frac{1}{n^{\prime}} H\left(\tilde{K} \mid \tilde{D}_{1}, Z^{B n^{\prime}}(s-1), h_{m}^{n}, h_{e}^{n}\right) \\
\stackrel{(b)}{\leq} & \frac{1}{n^{\prime}} H\left(\tilde{D}_{1} \oplus \tilde{K}\right)-\frac{1}{n^{\prime}} H\left(\tilde{K} \mid \tilde{D}_{1}, Z^{B n^{\prime}}(s-1), h_{m}^{n}, h_{e}^{n}\right) \\
\stackrel{(c)}{=} & R_{1}(\mathbf{h})-\frac{1}{n^{\prime}} H\left(\tilde{K} \mid Z^{B n^{\prime}}(s-1), h_{m}^{n}, h_{e}^{n}\right)
\end{aligned}
$$

where (a) follows from the conditional independence of $\tilde{D}_{1}$ on $Z^{n^{\prime}}$ and $D_{2}$ given $D_{1}$ and $Z^{B n^{\prime}}(s-1)$, (b) follows from the fact that conditioning does not increase entropy, and (c) follows from the uniform distribution of $\tilde{K}$ and the independence of $\tilde{K}$ and $\tilde{D}_{1}$ given $Z^{B n^{\prime}}(s-1)$.

Using the same argument as in (20) and (21), and from (18), it is straightforward to see

$$
\begin{aligned}
\frac{1}{n^{\prime}} H\left(\tilde{K} \mid Z^{B n^{\prime}}(s-1), h_{m}^{n}, h_{e}^{n}\right) & \geq \frac{1}{n^{\prime}} H(\tilde{K})-\epsilon \\
& =R_{1}(\mathbf{h})-\epsilon .
\end{aligned}
$$

Substituting this in (23) and (22), we get

$$
\frac{1}{n^{\prime}} H\left(\tilde{D}_{1} \mid D_{2}, Z^{n^{\prime}}, Z^{B n^{\prime}}(s-1), h_{m}^{n}, h_{e}^{n}\right) \geq \frac{1}{n^{\prime}} H\left(\tilde{D}_{1}\right)-\epsilon
$$


Finally, combining (19), (21), and (25) completes the proof.

In the previous result, the achievable rate satisfies the requirements given by the Definition 1. Consequently, the outage probability is zero with the proposed scheme. We also remark that, with the above achievability scheme, the initialization phase is over the first super-block, during which the data is not transmitted. With a simple modification, the data can also be transmitted during the first super-block by sacrificing the security of only the corresponding packets, which is negligibly small compared to the whole message.

The final step in this section is to establish the asymptotic optimality of the proposed security scheme in the high SNR regime. The following result achieves this objective by showing that the upper and lower bounds of Theorems 1 and 2 match in this asymptotic scenario for a wide class of invertible channels.

Lemma 3: In an asymptotic regime of high SNR, i.e., $\bar{P} \rightarrow \infty$, the delay limited secrecy capacity is given by

$$
\lim _{\bar{P} \rightarrow \infty} C_{s, d}^{F}=\mathbb{E}_{h_{m}>h_{e}}\left[\log \left(\frac{h_{m}}{h_{e}}\right)\right],
$$

assuming that $\mathbb{E}\left[\frac{1}{\min \left(h_{e}, h_{m}\right)}\right]$ is finite. Moreover, the capacity is achieved by the proposed one-time pad encryption scheme coupled with the key renewal process.

Proof: We only need to consider the lower bound as the right hand side of (26) is the ergodic secrecy capacity in the high SNR regime, which is by definition an upper bound on the delay limited secrecy capacity. To this end, in the proposed scheme, we set $q(\mathbf{h})=h_{e}$ resulting in $R_{2}(\mathbf{h})=0$. Furthermore, we let $P(\mathbf{h})=\frac{c}{\min \left(h_{e}, h_{m}\right)}$, where $c$ is a constant, which is chosen according to the average power constraint. The achievable rate expression in the high SNR regime is then given by

$$
\lim _{\bar{P} \rightarrow \infty} R_{s, d}^{F}=\lim _{\bar{P} \rightarrow \infty} \min _{\mathbf{h}} R_{1}(\mathbf{h}),
$$

where $R_{1}(\mathbf{h})$ is chosen to satisfy

$$
\begin{aligned}
\mathbb{E}\left[R_{1}(\mathbf{h})\right] & \leq \mathbb{E}\left[\left[\log \left(1+P(\mathbf{h}) h_{m}\right)-\log \left(1+P(\mathbf{h}) h_{e}\right)\right]^{+}\right] \\
R_{1}(\mathbf{h}) & \leq \log (1+c)
\end{aligned}
$$

As $\bar{P} \rightarrow \infty$, it is easy to see that $c \rightarrow \infty$ since $\mathbb{E}\left[\frac{1}{\min \left(h_{e}, h_{m}\right)}\right]$ is finite, implying that the second constraint in (28) is loose. Also, it is easy to see that the first constraint converges to the right hand side of the lemma. Then, by choosing $R_{1}(\mathbf{h})=\mathbb{E}_{h_{m}>h_{e}}\left[\log \left(\frac{h_{m}}{h_{e}}\right)\right]$, both constraints of (28) are satisfied and hence the result is proved.

\section{ONLY MAin ChANNEl CSI}

In this section we assume that only the legitimate receiver CSI is available at the transmitter. First, we have the following upper bound.

Theorem 4: The delay limited secrecy capacity when only the legitimate receiver channel state is available at the transmitter, $C_{s, d}^{M}$, is upper bounded by

$$
C_{s, d}^{M} \leq \max _{\substack{P\left(h_{m}\right) \\ \text { s.t. } \mathbb{E}\left[P\left(h_{m}\right)\right] \leq \bar{P}}} \min \left\{R_{s}^{M}, R_{d}^{M}\right\}
$$


where $R_{s}^{M}$ and $R_{d}^{M}$ are given as follows.

$$
\begin{aligned}
& R_{s}^{M}=\mathbb{E}\left[\log \left(1+P\left(h_{m}\right) h_{m}\right)-\log \left(1+P\left(h_{m}\right) h_{e}\right)\right]^{+} \\
& R_{d}^{M}=\min _{h_{m}} \log \left(1+P\left(h_{m}\right) h_{m}\right)
\end{aligned}
$$

Proof: The proof follows the same argument as that of Theorem 1 with the power control policy $P\left(h_{m}\right)$.

The achievability scheme in this scenario is different from the previous scenario in two key aspects: 1) the lack of knowledge about $h_{e}$ forces us to secure the whole delay sensitive traffic with the one time pad approach (i.e., setting the rate $R_{2}(\mathbf{h})$ to zero) and 2) the binning scheme of the key renewal process must now operate on the level of the super-block to average-out the fluctuations in $h_{e}$. On the other hand, the delay sensitive packet must be decoded after each block. This makes the use of the delay sensitive packet as a randomization signal a rather challenging task. Therefore, the achievable rate reported in the following result is obtained by superimposing the binning scheme (used to secure the key) on the delay limited traffic (secured by the key bits sent in the previous super-block).

Theorem 5: For any given arbitrarily small $\epsilon$, the $\epsilon$-delay limited secrecy capacity in the only main CSI scenario, $C_{s, d}^{M}(\epsilon)$, is lower bounded by the following $\epsilon$-achievable rate.

$$
C_{s, d}^{M}(\epsilon) \geq R_{s, d}^{M}(\epsilon)=\max _{\substack{P\left(h_{m}\right) \\ \text { s.t. } \mathbb{E}\left[P\left(h_{m}\right)\right] \leq \bar{P}}} \min \left\{R_{s}, R_{d}^{M}\right\},
$$

where

$$
\begin{aligned}
R_{s} & =\mathbb{E}\left[\log \left(1+P\left(h_{m}\right) h_{m}\right)-R_{s, d}^{M}(\epsilon)-\log \left(1+P\left(h_{m}\right) h_{e}\right)\right]^{+}, \\
R_{d}^{M} & =\min _{h_{m}} \log \left(1+P\left(h_{m}\right) h_{m}\right) .
\end{aligned}
$$

Proof: First, fix a power control policy $P\left(h_{m}\right)$. The achievable rate is then obtained by maximizing over all power control policies satisfying the average power constraint. We start by describing our scheme. We divide the channel uses into super-blocks and further divide each super-block into blocks such that the coherence interval is $n^{\prime}$ symbols as considered in the proof of Theorem 2. In this scenario, we utilize the achievable secrecy rate within a block only for the key generation. That is, data is transmitted only by using the one-time pad encryption in contrast to the scheme used in Theorem 2. Due to the lack of knowledge of $h_{e}$, the key is decoded at the end of each super-block whereas the data packets are still decoded block by block using the key sent in the previous super-block. A given message $W \in\left\{1,2, \cdots, 2^{n R_{s, d}^{M}(\epsilon)}\right\}$, is divided into $(S-1) B$ data packets, each represented by $\tilde{D}(s, b)$ for $s \in\{2, \cdots, S\}$ and $b \in\{1, \cdots, B\}$, where each packet is sent with rate $R_{s, d}^{M}(\epsilon)$ during the corresponding block of the channel. The data packet $\tilde{D}(s, b)$ is transmitted along with the generated key using the one-time pad scheme. Initial key generation and key renewal is similar to the scheme in Theorem 2. We remark that, similar to Theorem 2, the initialization phase duration becomes negligible as $S \rightarrow \infty$.

Codebook Generation and Encoding: Let $R=\min \left\{R_{s}, R_{d}^{M}\right\}$. For any given block $(s, b), s>1$, we use the $n^{\prime} R$ remaining bits from the key $K(s-1)$ and denote corresponding bits as $\tilde{K}(s, b)$. These bits are used in a 
one-time pad scheme to construct

$$
D(s, b)=\tilde{D}(s, b) \oplus \tilde{K}(s, b)
$$

The encrypted bits are then mapped to a message $w(s, b) \in\left\{1,2, \cdots, 2^{n^{\prime}} R\right\}$. For the key renewal process, the binning scheme is constructed over the super block $s$, as in the achievable scheme used in [2], such that the output bits of the encoder are divided into $B$ independent blocks each consists of $n^{\prime}\left[\log \left(1+P\left(h_{m}(s, b)\right) h_{m}(s, b)\right)-R-\epsilon\right]$ bits where $b \in\{1,2, \cdots, B\}$ is the coherence interval. We then combine those bits with the $n^{\prime} R$ reserved bits for the encrypted data packet and encode them using a member of the generated Gaussian codebook ensemble, which has $2^{n^{\prime}\left[\log \left(1+h_{m}(s, b) P\left(h_{m}(s, b)\right)\right)-\epsilon\right]}$ codewords. The channel input, denoted by $X^{n^{\prime}}(s, b)$, corresponding to the message from the code is sent from the transmitter.

Error Analysis: Each codeword is decoded at the end of the block releasing the delay sensitive packet. Following the same argument used in proof of Theorem $2, P_{e}^{n^{\prime}}(s, b)$ can be made arbitrarily small as $n^{\prime} \rightarrow \infty$ for each $(s, b)$. The key bits are decoded at the end of the binning codeword (i.e., super block) following the same argument used in [2]. Therefore, as $n^{\prime} \rightarrow \infty$ and $B \rightarrow \infty$, the proposed key rate is achievable, where the encrypted data bits are not used as a part of the randomization message.

Equivocation Computation: We will show that, for the given $\epsilon$ (can be arbitrarily small) and for any given $\epsilon^{\prime}>0$,

$$
P_{\text {out }}\left(s, b, R_{s, d}(\epsilon), \epsilon^{\prime}\right) \leq \epsilon
$$

with the proposed scheme. The equivocation per block for arbitrary chosen block $(s, b)$ is given by

$$
\begin{aligned}
n^{\prime} R_{e}(s, b) & =H\left(\tilde{D}(s, b) \mid Z^{n}, h_{m}^{n}, h_{e}^{n}\right) \\
& =H\left(\tilde{D} \mid Z^{n^{\prime}}(1,1), Z^{n^{\prime}}(1,2), \cdots, Z^{n^{\prime}}(S, B), h_{m}^{n}, h_{e}^{n}\right) \\
& \stackrel{(a)}{=} H\left(\tilde{D} \mid Z^{n^{\prime}}(s, b), Z^{B n^{\prime}}(s-1), h_{m}^{n}, h_{e}^{n}\right) \\
& =H\left(\tilde{D} \mid Z^{B n^{\prime}}(s-1), h_{m}^{n}, h_{e}^{n}\right)-I\left(\tilde{D} ; Z^{n^{\prime}} \mid Z^{B n^{\prime}}(s-1), h_{m}^{n}, h_{e}^{n}\right) \\
& \stackrel{(b)}{=} H\left(\tilde{D} \mid h_{m}^{n}, h_{e}^{n}\right)-I\left(\tilde{D} ; Z^{n^{\prime}} \mid Z^{B n^{\prime}}(s-1), h_{m}^{n}, h_{e}^{n}\right) \\
& \geq H\left(\tilde{D} \mid h_{m}^{n}, h_{e}^{n}\right)-I\left(\tilde{D} ; Z^{n^{\prime}}, D \mid Z^{B n^{\prime}}(s-1), h_{m}^{n}, h_{e}^{n}\right) \\
& =H\left(\tilde{D} \mid h_{m}^{n}, h_{e}^{n}\right)-H\left(\tilde{D} \mid Z^{B n^{\prime}}(s-1), h_{m}^{n}, h_{e}^{n}\right)+H\left(\tilde{D} \mid Z^{n^{\prime}}, D, Z^{B n^{\prime}}(s-1), h_{m}^{n}, h_{e}^{n}\right) \\
& \stackrel{(c)}{=} H\left(\tilde{D} \mid h_{m}^{n}, h_{e}^{n}\right)-H\left(\tilde{D} \mid Z^{B n^{\prime}}(s-1), h_{m}^{n}, h_{e}^{n}\right)+H\left(\tilde{D} \mid D, Z^{B n^{\prime}}(s-1), h_{m}^{n}, h_{e}^{n}\right) \\
& =H\left(\tilde{D} \mid h_{m}^{n}, h_{e}^{n}\right)-I\left(\tilde{D} ; D \mid Z^{B n^{\prime}}(s-1), h_{m}^{n}, h_{e}^{n}\right) \\
& =H\left(\tilde{D} \mid h_{m}^{n}, h_{e}^{n}\right)-H\left(D \mid Z^{B n^{\prime}}(s-1), h_{m}^{n}, h_{e}^{n}\right)+H\left(D \mid \tilde{D}, Z^{B n^{\prime}}(s-1), h_{m}^{n}, h_{e}^{n}\right) \\
\geq & H\left(\tilde{D} \mid h_{m}^{n}, h_{e}^{n}\right)-H(\tilde{D} \oplus \tilde{K})+H\left(\tilde{K} \mid \tilde{D}, Z^{B n^{\prime}}(s-1), h_{m}^{n}, h_{e}^{n}\right) \\
& \stackrel{(d)}{=} H\left(\tilde{D} \mid h_{m}^{n}, h_{e}^{n}\right)-H(\tilde{D} \oplus \tilde{K})+H\left(\tilde{K} \mid Z^{B n^{\prime}}(s-1), h_{m}^{n}, h_{e}^{n}\right)
\end{aligned}
$$


where $Z^{B n^{\prime}}(s-1)=Z^{n^{\prime}}(s-1,1), Z^{n^{\prime}}(s-1,2), \cdots, Z^{n^{\prime}}(s-1, B)$ is the output of the channel in the previous super-block $s-1$, the index $(s, b)$ is omitted in $\tilde{D}(s, b)$ and $\tilde{K}(s, b)$, (a) follows from the independence between block $(s, b)$ and other transmissions, (b) follows from the independence of $\tilde{D}$ and $Z^{B n^{\prime}}(s-1)$, (c) follows from the independence between $\tilde{D}$ and $Z^{n^{\prime}}$ given $D$ and $Z^{B n^{\prime}}(s-1)$, and (d) follows from the independence of $\tilde{K}$ and $\tilde{D}$ given $Z^{B n^{\prime}}(s-1)$.

It remains to bound the last term in (34). Here, if we satisfy

$$
\frac{1}{n^{\prime}} H\left(\tilde{K} \mid Z^{B n^{\prime}}, h_{m}^{n}, h_{e}^{n}\right) \geq \frac{1}{n^{\prime}} H(\tilde{K})-\epsilon^{\prime}
$$

we have, from (34), that

$$
R_{e}(s, b) \geq R_{s, d}-\epsilon^{\prime}
$$

Therefore, the secrecy outage event happens once (35) is not satisfied with the given $\epsilon^{\prime}$. We denote this event as follows.

$$
\mathcal{O}\left(\epsilon^{\prime}\right) \triangleq\left\{\frac{1}{n^{\prime}} H\left(\tilde{K} \mid Z^{B n^{\prime}}, h_{m}^{n}, h_{e}^{n}\right)<\frac{1}{n^{\prime}} H(\tilde{K})-\epsilon^{\prime}\right\}
$$

Consequently, we will use the bound

$$
P_{\text {out }}\left(s, b, R_{s, d}(\epsilon), \epsilon^{\prime}\right) \leq \operatorname{Pr}\left\{\mathcal{O}\left(\epsilon^{\prime}\right)\right\}
$$

in order to show that the outage probability can be made less than $\epsilon$.

Following the argument given in [2], one can see that the following key rate can be achieved with perfect secrecy (as $n^{\prime} \rightarrow \infty$ and $B \rightarrow \infty$ ).

$$
R_{s}=\mathbb{E}\left[\log \left(1+P\left(h_{m}\right) h_{m}\right)-R-\log \left(1+P\left(h_{m}\right) h_{e}\right)\right]^{+}
$$

with

$$
\frac{1}{n^{\prime} B} H\left(K(s-1) \mid Z^{B n^{\prime}}(s-1), h_{m}^{n}, h_{e}^{n}\right) \geq \frac{1}{n^{\prime} B} H(K(s-1))-\epsilon_{1}
$$

where $Z^{B n^{\prime}}(s-1)$ is the received signal by Eve for the super-block $s-1$ and $\epsilon_{1}>0$ is arbitrarily small as $n^{\prime}, B \rightarrow \infty$. Here, we denote the number of blocks within the super block $s-1$ for which the event $\mathcal{O}\left(\epsilon^{\prime}\right)$ holds as $\beta$. Then, from (36) and (38), we conclude that $n^{\prime} \epsilon^{\prime} \beta \leq n^{\prime} B \epsilon_{1}$, which further implies

$$
\operatorname{Pr}\left\{\mathcal{O}\left(\epsilon^{\prime}\right)\right\}=\lim _{B \rightarrow \infty} \frac{\beta}{B} \leq \lim _{B \rightarrow \infty} \frac{\epsilon_{1}}{\epsilon^{\prime}} .
$$

At this point, as $\epsilon_{1}$ can be arbitrarily made small as $n^{\prime}, B \rightarrow \infty$, we conclude from (36) that, for any given arbitrarily small $\epsilon^{\prime}$ and $\epsilon$

$$
P_{\text {out }}\left(s, b, R_{s, d}(\epsilon), \epsilon^{\prime}\right) \leq \epsilon
$$

for sufficiently large $n^{\prime}$ and $B$.

We note that, when only the main CSI is available, we followed the $\epsilon$-achievability notion given by definition 3 with some arbitrarily small $\epsilon$, which means that the above claimed rate is achievable with perfect secrecy for every realization of the channel except for a subset whose probability can be arbitrarily made small. 
Finally, we report numerical results that validate our theoretical claims. In the full CSI case, we set $q(\mathbf{h})=h_{e}$ (hence $R_{2}(\mathbf{h})=0$ ) and use channel inversion power control policy for the achievable rate. We first set both $h_{m}$ and $h_{e}$ to be independent and identically distributed Chi-Square random variables with four degrees of freedom. Remarkably, as shown in the upper two curves in Fig. 2, even with these, in general suboptimal, choices of $q(\mathbf{h})$ and $P(\mathbf{h})$, the lower and upper bounds coincide in the high SNR regime. The same trend is observed in the lower two curves of the figure corresponding to the case with $\mathbb{E}\left[h_{e}\right]=2 \mathbb{E}\left[h_{m}\right]$. Figure 3 corresponds to the case where only the main channel CSI is available at the transmitter. Here, the channel inversion power control policy is used for both the upper and lower bounds. The achievability of a non-zero delay limited rate is evident even for the case in which the eavesdropper channel is better than the main channel on the average.

\section{CONCLUSIONS}

We have studied the delay limited secrecy capacity of the slow-fading channel under different assumptions on the transmitter CSI. Our achievability arguments are based on a novel two-stage scheme that allows for overcoming the secrecy outage phenomenon for a wide class of channels. The scheme is based on sharing a delay tolerant private key, using random binning, and then using the key to encrypt the the delay sensitive packets in a one time pad format. For the full CSI case, our scheme is shown to be asymptotically optimal, i.e., at high SNR regime, for many relevant channel distributions. When only the main channel CSI is available, the two-stage scheme achieves a non-zero delay-limited secure rate, with high probability, for invertible channels. Finally, one can easily identify several avenues for future works. For example, 1) obtaining sharp capacity results for finite values of SNR, 2) extending the results to multiuser scenarios, 3) characterizing the optimal power control policies, and 4) extending the framework to bursty traffic by allowing for buffer delays.

\section{REFERENCES}

[1] A. D. Wyner, “The Wire-Tap Channel,” The Bell System Technical Journal, vol. 54, no. 8, pp. 1355-1387, Oct. 1975.

[2] P. K. Gopala, L. Lai, and H. El Gamal, "On the Secrecy Capacity of Fading Channels," IEEE Trans. on Inf. Theory, vol. 54, no. 10, pp. 4687-4698, Oct. 2008.

[3] S. V. Hanly and D. N. C. Tse "Multiaccess Fading Channels-Part II: Delay-Limited Capacities," IEEE Trans. on Inf. Theory, vol. 44, no. 7, pp. 2816-2831, Nov. 1998.

[4] R. A. Berry and R. G. Gallager, "Communication Over Fading Channels with Delay Constraints," IEEE Trans. on Inf. Theory, vol. 48, no. 5, pp. 1135-1149, May 2002.

[5] J. Lee and N. Jindal, "Energy-efficient Scheduling of Delay Constrained Traffic over Fading Channels," IEEE Trans. on Wireless Communications, to appear.

[6] M. Bloch, J. Barros, M. R. D. Rodrigues, and S. W. McLaughlin, "Wireless Information-Theoretic Security," IEEE Trans. on Inf. Theory, volume 54, no. 6, pp. 2515-2534, June 2008.

[7] G. S. Vernam, "Cipher Printing Telegraph Systems For Secret Wire and Radio Telegraphic Communications," J. Amer. Inst. Elect. Eng., vol. 55, pp. 109-115, 1926.

[8] C. E. Shannon, "Communication Theory of Secrecy Systems," The Bell System Technical Journal, vol. 28, pp. 656-715, Oct. 1949.

[9] G. Caire, G. Taricco and E. Biglieri, "Optimal Power Control Over Fading Channels," IEEE Trans. on Inf. Theory, vol. 45, no. 5, pp. 1468-1489, July 1999. 
[10] L. Ozarow, S. Shamai (Shitz), and A. Wyner, "Information theoretic considerations for cellular mobile radio," IEEE Trans. Veh. Technol., vol. 43, no. 2, pp. 359-378, May 1994.

[11] V. M. Prabhakaran, K. Eswaran, and K. Ramchandran, "Secrecy via Sources and Channels - A Secret Key - Secret Message Rate Trade-off Region," in Proc. of IEEE International Symposium on Information Theory (ISIT 2008), Toronto, ON, Canada, July 2008.

[12] T. Cover and J. Thomas, "Elements of Information Theory," Second Edition, John Wiley and Sons, Inc., 2006.

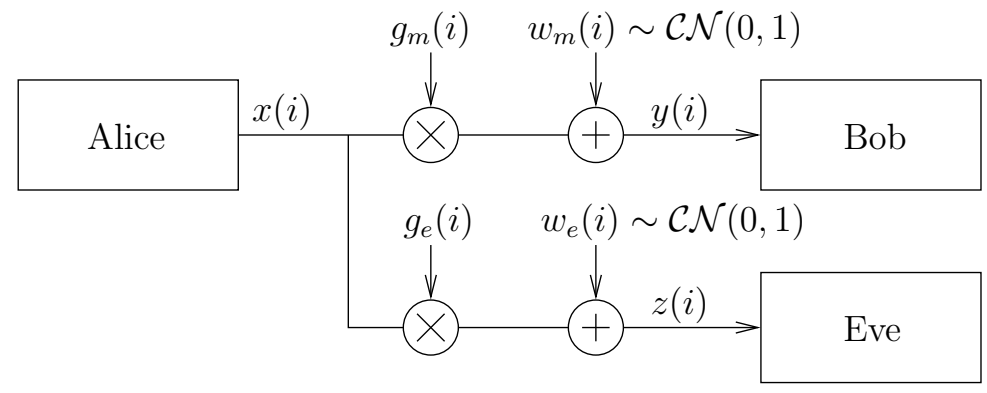

Fig. 1. System Model.

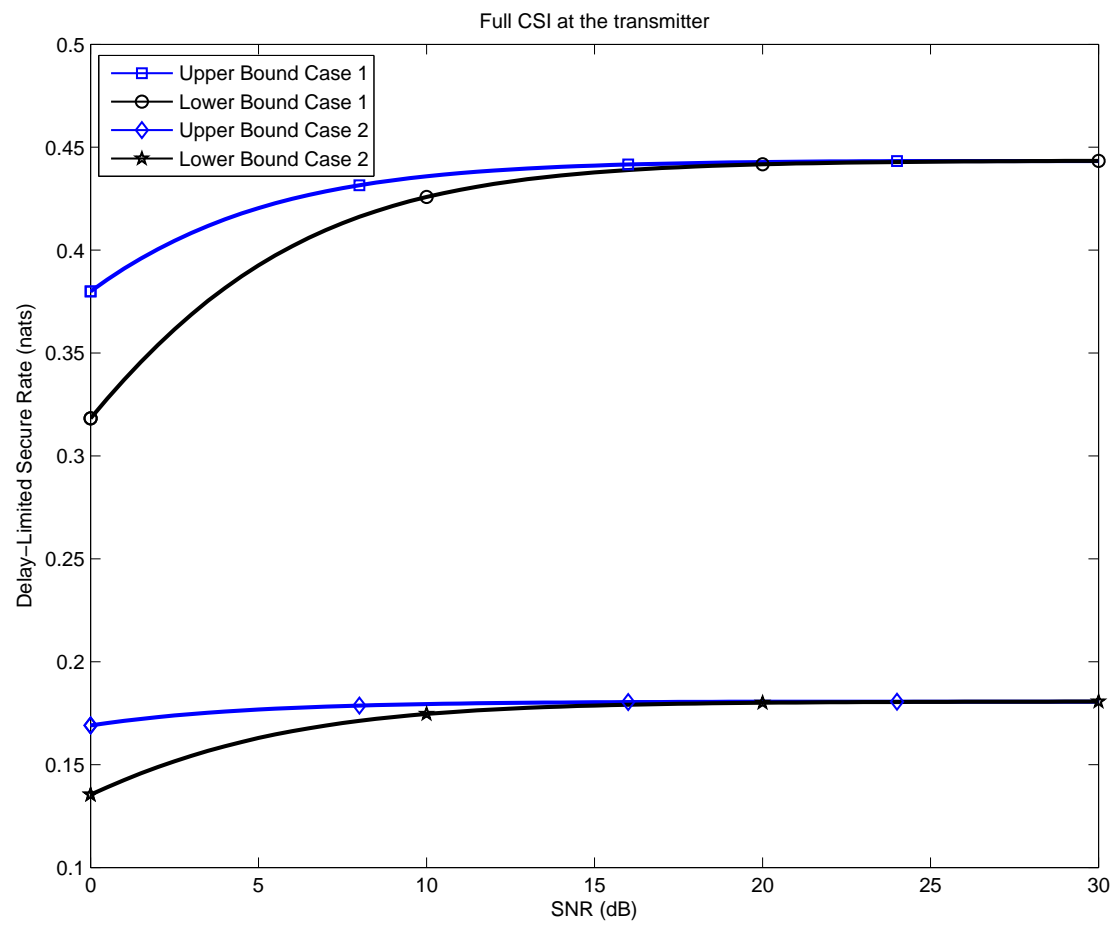

Fig. 2. Simulation results for the Full CSI. $\mathbb{E}\left[h_{e}\right]=\mathbb{E}\left[h_{m}\right]$ in case 1 , and $\mathbb{E}\left[h_{e}\right]=2 \mathbb{E}\left[h_{m}\right]$ in case 2 . 


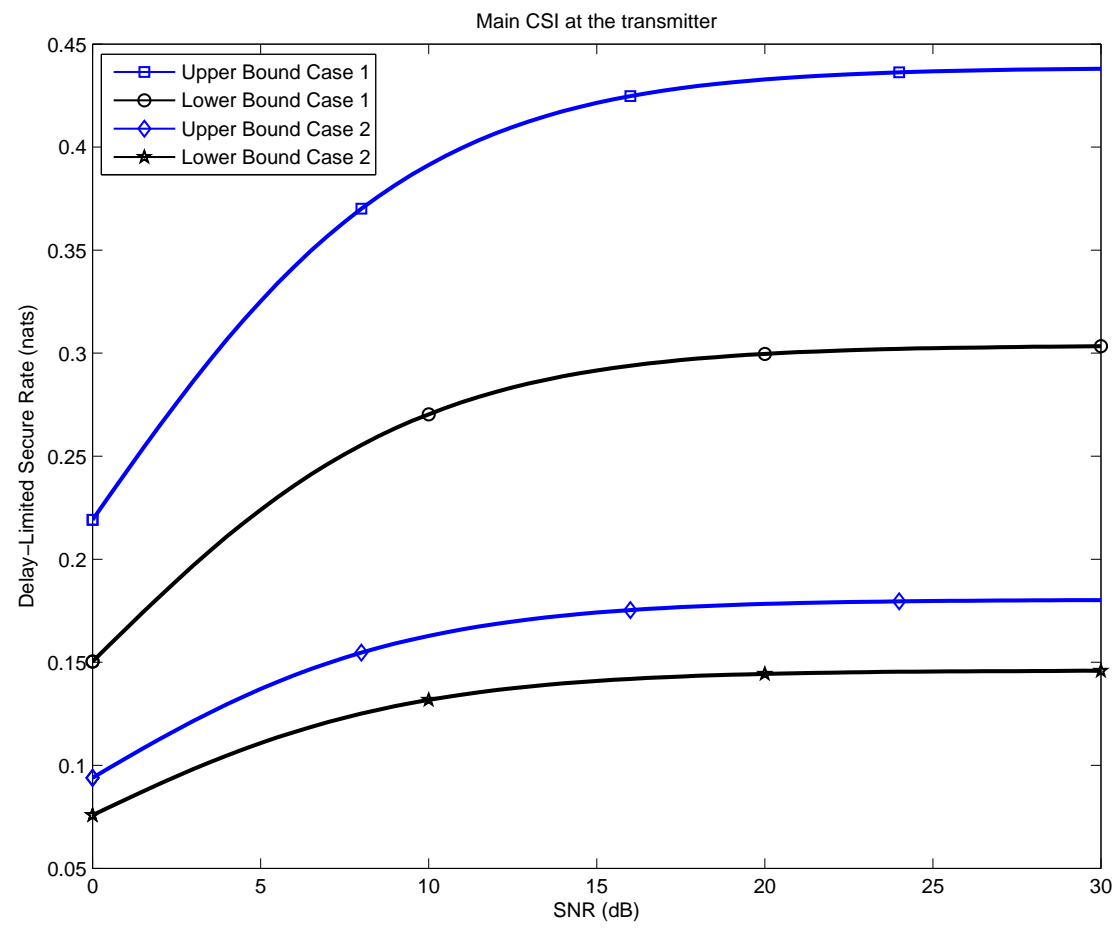

Fig. 3. Simulation results for the Main CSI. $\mathbb{E}\left[h_{e}\right]=\mathbb{E}\left[h_{m}\right]$ in case 1 , and $\mathbb{E}\left[h_{e}\right]=2 \mathbb{E}\left[h_{m}\right]$ in case 2 . 